\title{
Unmanned Ground Vehicle Equipped With Ground Penetrating Radar FOR IMPROVISED EXPLOSIVES DETECTION
}

Submitted: $15^{\text {th }}$ July 2021; accepted: $20^{\text {th }}$ September 2021

\author{
Piotr Szynkarczyk, Józef Wrona, Mateusz Pasternak, Arkadiusz Rubiec, Piotr Serafin
}

DOI: 10.14313/JAMRIS/2-2021/10

\begin{abstract}
:
The article presents some objectives and results of the European Defense Agency Program on: Improvised Explosive Devices Detection (IEDDET Program). The goal of the article is to describe the work, results and recommendations regarding Unmanned Ground Vehicle (UGV) and Ground Penetrating Radar (GPR) - contributions within the MUSICODE project. Its scope and goals were presented, which are in line with the objectives of the IEDDET Program taking into consideration that the recommendations (for UGV and GPR) are related to the goals, not the results of MUSICODE project. There were described scenarios and the resulting changes in the structure implemented in the UGV - the FLORIAN robot which served as a sensors carrier including Ground Penetrating Radar (GPR). The main focus of the article is to find the answer to the research question: what is an impact of using the GPR to be mounted on the UGV to detect improvised explosive devices (IEDs) on the UGV construction and the GPR results. The structure of this radar was described and examples of tests results were presented. The summary presents recommendations for the construction of an unmanned land platform to carry sensors used in the work carried out in the MUSICODE project and conclusions regarding GPR, resulting from the experiences gained under the IEDDET Program.
\end{abstract}

Keywords: Improvised Explosive Devices Detection, Unmanned Ground Vehicle, Ground Penetrating Radar

\section{Introduction}

The goal of the IEDDET program was to develop and demonstrate multi-sensor detection systems for detecting IEDs. As a result, it will enable the increase of the ability of the army to move quickly and safely on roads and off-road. Representatives of five countries participated in the IEDDET program: Austria, Belgium, the Netherlands, Norway and Poland. According to the schedule, the program has been completed in the beginning 2020 but the final demo (in the form of a symposium without all heavy equipment present) that was planned to be hosted in Austria in March 2020 was postponed to September 2020 due to the Covid-19 situation [18].

The program included the implementation of three projects. Each of the three projects concerned different stages of IEDs detection: early warning, remote detec- tion as well as confirmation and identification (Fig. 1). The key issue was to improve overall capability by exchanging information between the three phases. Different requirements of each of the three phases mean that a separate project was launched to implement each of them under the program (Fig. 1):

- early warning - Vehicle Mounted Early Warning of Indirect Indicators of IEDs (VMEWI3);

- confirmation and identification - Confirmation, Identification and Airborne Early Warning of IEDs (CONFIDENT);

- remote detection - UGV stand-off multi-sensor platform for IED component detection (MUSICODE).

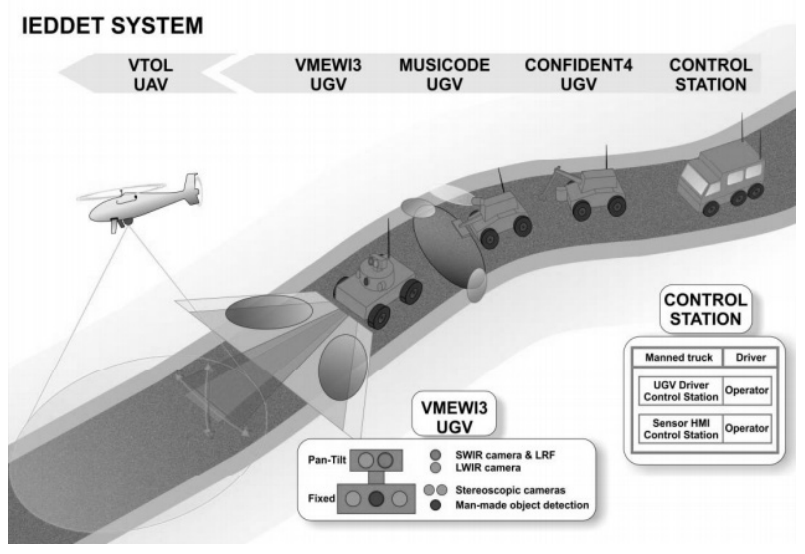

Fig. 1. The IEDDET Programme - the components of IEDDET System [17]

The goal of the VMEWI3 project was to detect indirect IEDs presence indicators using cameras that observe the area in front of a moving vehicle. The project was managed by the Netherlands Organisation for Applied Scientific Research (Nederlandse Organisatie voor Toegepast-Natuurwetenschappelijk Onderzoek, TNO) in cooperation with industry - medium and small enterprises, so-called SME, universities and research institutions. This group of entities included: The Armament and Defense Technology Agency (ARWT) from Austria, Royal Military Academy (RMA) from Belgium, Nederlandse Instrumenten Compagnie (Nedinsco), ViNotion BV, Technische Universiteit Eindhoven (TU/e), Quest Photonic Devices BV (Quest) from the Netherlands, PCO S.A. and the Military University of Technology, both entities from Poland. In the framework of the project there was developed and tested a system consisting of an Unmanned Ground Platform (UGV) with an optical multi-sensor IEDs early warning system. 
This CONFIDENT project primarily aims were to confirm the detection and identification of significant IED components, including electronic components, explosives, chemical, biological, radiological and nuclear (CBRN) weapon and to provide complementary early warning capability. The project was carried out by a consortium led by the Armament and Defense Technology Agency (ARWT) from Austria, Royal Military Academy (RMA) from Belgium, Military University of Technology from Poland and The Norwegian Defence Research Establishment (Forsvarets forskningsinstitutt, FFl from Norway. The technology demonstrators were mounted on remotely controlled platforms: land (UGV) and flying (UAV). The problem of how to use a given set of possibly heterogeneous unmanned aerial vehicles (UAVs) to provide protection to a moving convoy of ground vehicles was also in the interest of some scientists [4].

The MUSICODE project developed a multi-sensor remote detection system for IED components mounted on the Polish unmanned ground vehicle - FLORIAN, which is the final product of the Polish project - INNOTECH-K1/IN1/70/154619/NCBiR/12, developed by the Consortium consisting of: WB Electronics SA, HYDROMEGA Sp. z o.o. and the Military University of Technology. The MUSICODE project was coordinated by The Norwegian Defence Research Establishment (Forsvarets forskningsinstitutt, FFI) from Norway. The members of consortia were: the Armament and Defense Technology Agency (ARWT) from Austria, Royal Military Academy (RMA) from Belgium, the Netherlands Organisation for Applied Scientific Research (Nederlandse Organisatie voor Toegepast - Natuurwetenschappelijk Onderzoek, TNO), ŁUKASIEWICZ Research Network - Industrial Research Institute for Automation and Measurements PIAP, Military Institute of Technical Engineering (WITI), Consortia Electronica (Cons-el), Advanced Protection Systems (APS), Military University of Technology. The system developed in the project analyzed data coming from sensors scanning the space in front of the UGV - on the ground and in the ground. The tasks within the MUSICODE project were also performed using data coming from the VMEWI3 system. The Ground Penetrating Radar (GPR), one of the sensors that detected items in the ground and the FLORIAN - Unmanned Ground Vehicle (UGV) are the main subjects of the article to discuss and find the answer to the research question: what is an impact of using the GPR to be mounted on the UGV to detect improvised explosive devices (IEDs) on the UGV construction and the GPR results. There was used the control vehicle (CV) (Fig. 2) equipped with a control station (Fig. 1).

There were some efforts in the area of MUSICODE project. In [3] there is presented a limited, operationally-focused overview of the current status of detection technologies to be focused on those technologies that directly detect the explosives, as opposed to those that detect secondary properties of the threat, such as the casing, associated wires or electronics.

But one of the key issues in this area is detection of explosives. The design and fabrication process for an electrochemical - "electronic nose" type sensor to detect explosives is in the interest of some scientists [5]. In [6] the novel Ground Penetrating Radar (GPR) system to detect Improvised Explosive Devices (IEDs) was numerically evaluated that is composed of a transmitter placed on a vehicle and looking forward and a receiver mounted on a drone and looking downwards [6]. In literature it is possible to find not only military context of the usage of UGVs. In [7], the results of research on the identification of gaps in the current state-of-the-art that impede the use of UGVs in various scenarios are presented. The challenge of developing infrastructure with the use of UGVs has common aspects in both civilian and military areas. The article [7] presents the results of work that show the influence of UGV Operational Requirements (ORs) on technological aspects of autonomous operation and on UGV Human-Machine Interfaces (HMIs). The authors have based the presented approach on previous experiences in analyzing UGV operations in security and military contexts, where they are widely applied.

There were some scientific activities in the world to be focused on the design and implementation of unmanned ground vehicle (UGV) for security and defense applications $[8,9,10]$. In [11], the concept of implementation of components of the remote control system to transform the main battle tank T-72 into Unmanned Ground Vehicle to perform mine clearance tasks and when crossing the river on its bottom, and some results of research of the system have been presented.

\section{Research Goals and Methodology of the MUSICODE Project}

The capability needs defined by the militaries inspired scientists to fulfill the Operational Requirements. Analysis of these requirements was the first step within the process to describe the intended use of the instrument to close the capabilities gap. Scenarios were described and functional and environmental requirements were derived from the scenarios. The requirements were defined to the demonstrator of technology to be possible to describe the anticipated future Route Clearance CONOPs (Concept of Operations) to discuss this issue with militaries [1].

The sensor systems and the UGV driving at an envisioned speed of $30 \mathrm{~km} / \mathrm{h}$ generated a high demand in the wireless communication and such a speed was the one of the goals not the result of the project. A high data rate, large range and short delay time were crucial for efficient and safe operation. For this reason, a new control communication system was developed. The developed system includes independent channels for UGV control, environment visualization and for sensor data transmission. The main issues to solve were the required high speed of data transmission under the constraints of limited frequency bands, short delay time and EMC/EMI due to limited antenna space [1].

Related to this some modifications were required to the manned control vehicle (CV). The control vehi- 


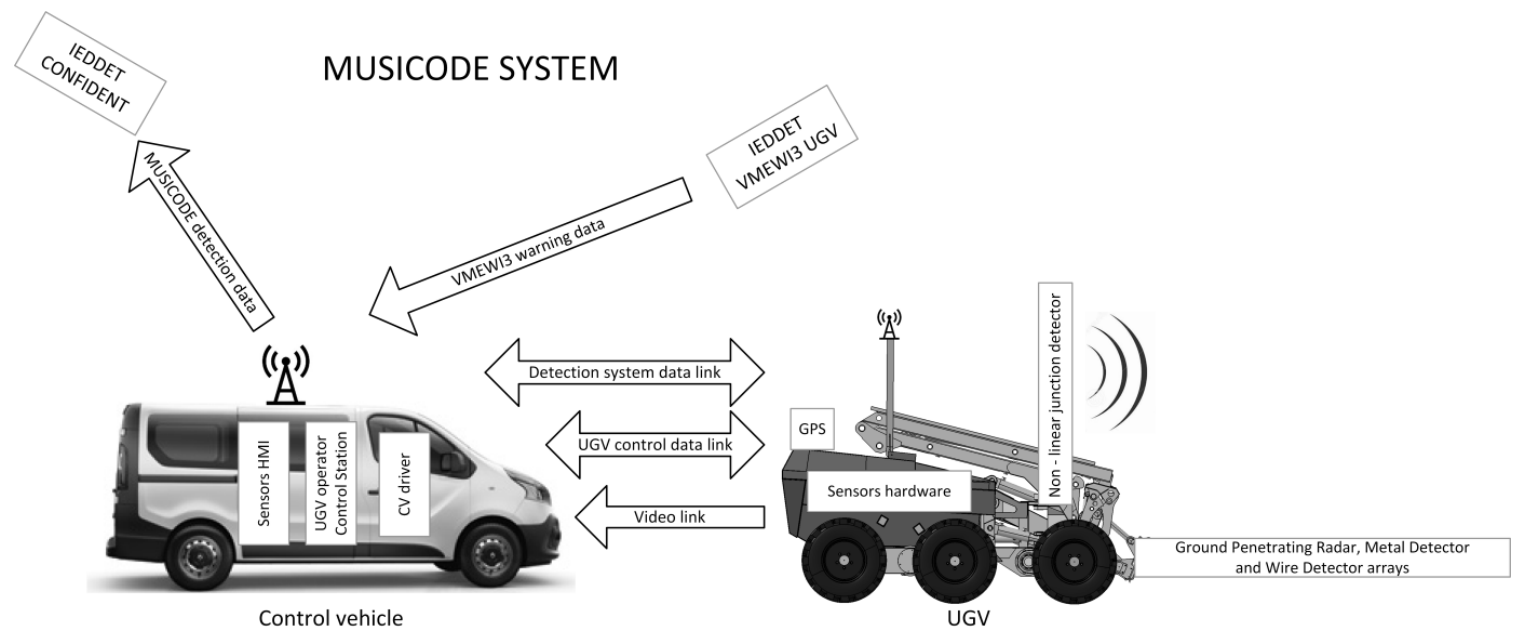

Fig. 2. The MUSICODE project concept - the components of MUSICODE System

cle needed to be fitted with a control station (Fig. 1) for the UGV, a control station for remote sensor operation, a power source and all the necessary antenna systems. Since the UGV and sensors have to be operated at high efficiency while the control vehicle is moving, care has to be taken for comfort and ergonomics of the operating crew and the testing personnel. For this reason, a 3D model was developed and tested including all supporting frames and cable placements [1].

Finally, all parts have to be integrated into one working system. This includes the control vehicle (CV), the wireless communication between the control vehicle and the UGV, the UGV remote control and terrain visualization system and the sensor control and data acquisition (Fig. 2,3). The second part of this task involved testing and debugging of the integrated system [1].

The general aim of the MUSICODE project was to develop and demonstrate a multi-sensor UGV for stand-off detection of IED components, where standoff as a result of realism was defined as distance to personnel, not to the sensors themselves [18].

The specific goals of the MUSICODE project were:

a) to evaluate and demonstrate state-of-the-art sensors of the following types: (i) downward looking Ground Penetration Radar (GPR), (ii) metal detector, (iii) command wire detector and (iv) non-linear junction detector. The sensors can be partly new developments, and partly improvement of off-the-shelf sensors;

b) to implement improved algorithms for signal processing and automated detection, both for individual sensors and in combination, and to present the results in a novel human-machine interface to the operator;

c) to exploit available information from the early warning phase by reading data from an off-line common detection map / database, and to pass information to the same off-line database for use of information in the following identification and confirmation phase; d) to integrate the multi-sensor suite on an adapted UGV system for participation in joint test and evaluation trials.

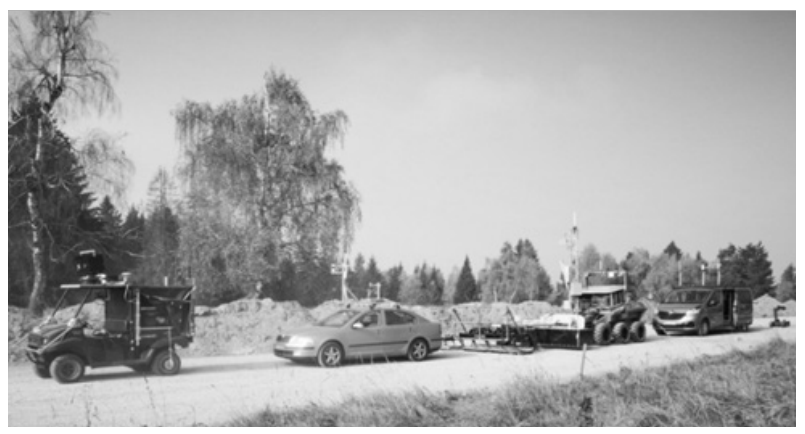

Fig. 3. View of some components of VMEWI3 and MUSICODE projects

The outcome of the project is at the $5^{\text {th }}$ Technology Readiness Level (TRL) - technology demonstrator for multi-sensor platform for IED component detection. There is a need to distinguish between the Project's goals and its results, which in some cases are not the same.

\section{The FLORIAN Platform Construction - Description of Changes Adapting the Platform for the Implementation of Tasks Within the MUSICODE Project}

The Unmanned Ground Vehicle (UGV) was the element merging the developed sensors into a IED detection system. Manned vehicle was used as a control vehicle (CV) to control UGV and sensors (Fig. 2) and to cooperate with control station (Fig. 1).

The UGV used in the MUSICODE project was developed as a part of a national research program as a robot for heavy rescue tasks. As a result, it was directly used in the MUSICODE project. To fulfill the detailed specifications some modifications of the UGV were required. In its original configuration the UGV had two attachments - the manipulator and the 
loader attachments. Their load capacities are $200 \mathrm{~kg}$ for manipulator and $1000 \mathrm{~kg}$ for loader attachment (with forklift). In the original version the UGV was controlled by a laptop plugged into the control console (Fig. 4b) [2].

The work conducted in the MUSICODE project identified several areas of the UGV which had to be modified. During the design development a particular focus was on Electromagnetic Compatibility (EMC) issues. There was defined the concept of reducing Electromagnetic Interferences (EMI) and the other works were done in the MUSICODE project required efforts in two areas:

- UGV adaptation;

- car adaptation for the role of the CV (Fig. 2).

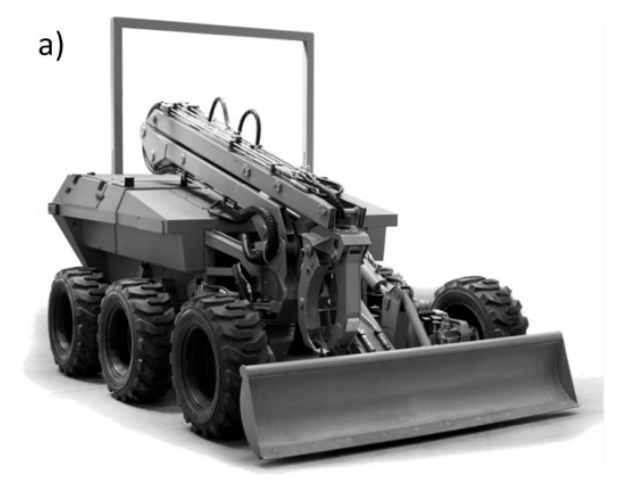

b)

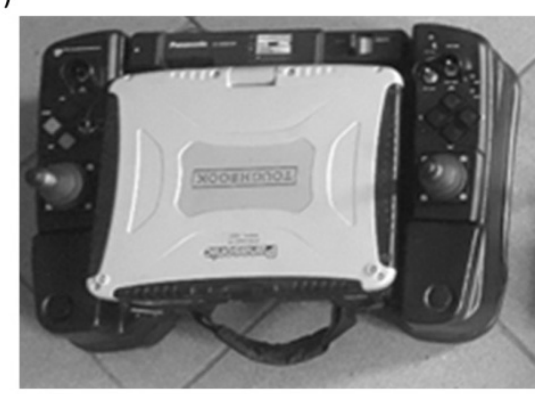

Fig. 4. View of the original version of the Florian platform: a) the Unmanned Ground Vehicle, b) the control console

The general adaptation works included:

- development of the supporting frame for the needs of the GPR, MD and WD mounting (Military Institute of Technical Engineering, WITI area of responsibility);

- development of the modification of the coupling system of the sensors frame with the UGV;

- development of nonlinear junction detection (NLJD) system fixing (Nederlandse Organisatie voor Toegepast Natuurwetenschappelijk Onderzoek, TNO area of responsibility);

- development of the fixing elements of the computers and fixing elements of the processing measurement data system;

- development of a power system for detection equipment;

- modification of the UGV engine chamber cooling system;
- development of a length measurement system for the UGV front suspension cylinders;

- development of positioning system;

- development of a data link;

- development of a video link;

- development of the UGV control software modification.

The vehicle adaptation to be a control vehicle (CV) (Fig. 2) mainly included:

- development of the UGV control station;

- development of an operation station for sensors Human Machine Interface(HMI) to operate with the detection systems (Forsvaretsforskningsinstitutt,FFI area of responsibility).

At the very design stage, the supporting frame element did not foresee any noteworthy UGV modifications. The only modification was a quick-action coupling which would connect the UGV to the frame. The mechanical element integrating the frame and the UGV is a metal attachment connected to a mounting plate on the UGV. This is the only function of the mounting frame, meaning that the UGV design modification on the support frame was indirect (Fig. 5) [2].

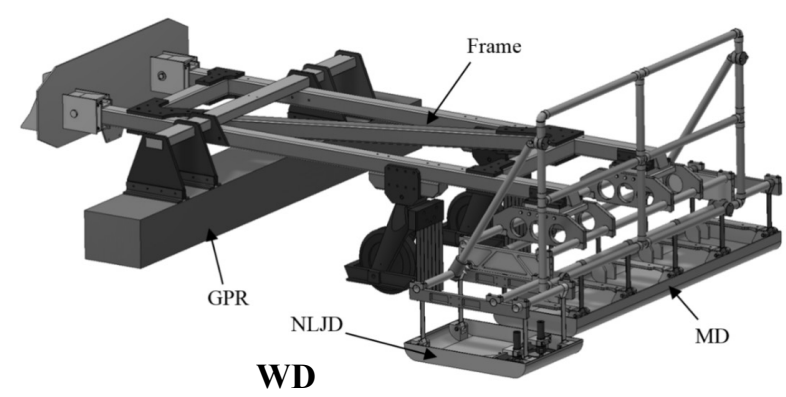

Fig. 5. Frame model with elements of the detection system: GPR - Ground Penetrating Radar, WD - Wire Detector, MD - Metal Detector

In the final version of the support frame, the position of the longitudinal reinforcement beam was modified and closing elements of the open square profiles used were added.

The change in the position of the longitudinal beam was determined by the weight of wire detector (WD) in order to achieve its stable operation while driving (Fig. 2, 5). The consequence of such loading was a greater vertical displacement of the corner of the frame and consequently a larger value of the torsion angle [2].

The change in the method of supporting the elements built on the support frame for MD, WD and the GPR (piano wheels) in relation to standard mounted tools resulted in the need to modify the existing locking system in the mechanical quick coupler.

The UGV of the MUSICODE project in the original version (Fig. 4a) was equipped with a standardized type of mechanical quick coupler in accordance with SAEJ2513. This design is dedicated to the working tools of skid steer loaders. The upper edge of the subplate is shaped in a triangular form, thanks to which it adapts to the fastening elements of the working tool. 
The final "locking" and "unlocking" of the work tool on the quick coupler occurs after turning the two levers and sliding the bolts from the bottom edge of the plate (Fig. 6) [2].

A.

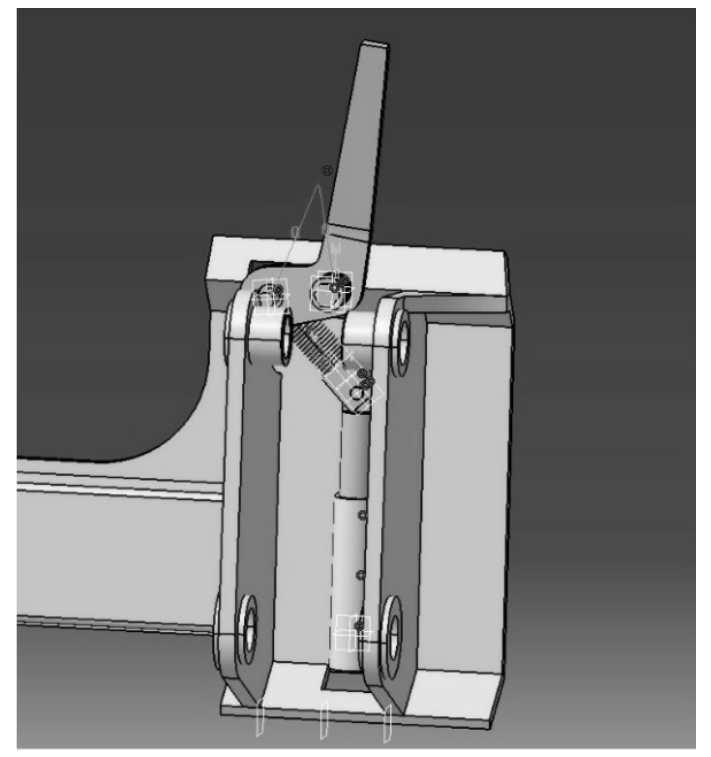

B.

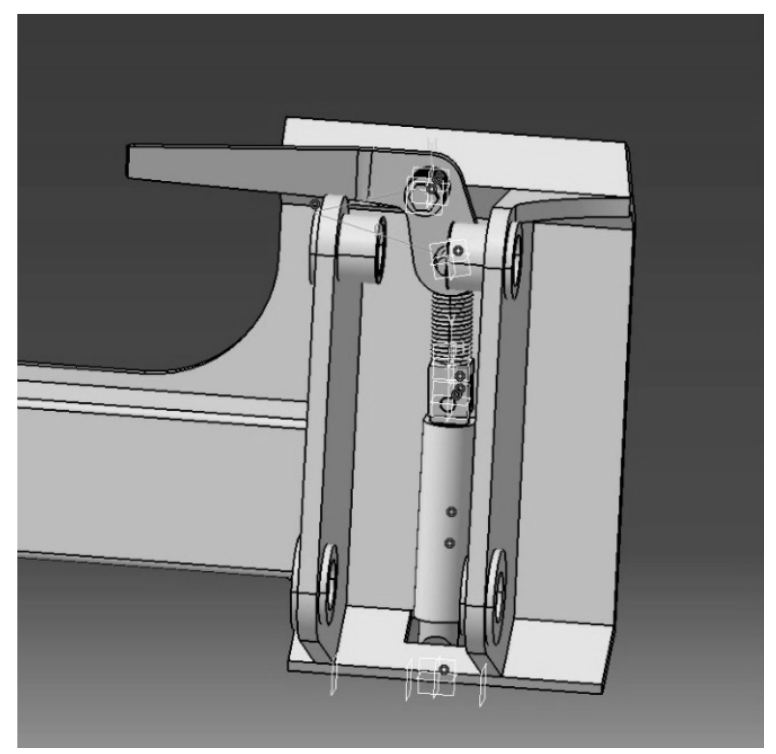

Fig. 6. Locking mechanism of the UGV quick coupler plate: A - unlocked position, B - locked position

The originally mounted tool on the UGV (Fig. 4) was loaded with a moment of force that prevented disconnection of the tool from the UGV thanks to quick coupler. For testing purposes the original tool was removed from the base platform. In its place a new version quick coupler plate was mounted (Fig. 7). The sensors frame was attached to the base platform's quick coupler plate using locking mechanism (Fig. 6). The attached sensors frame was equipped with two road wheels that play a supporting role. In this case, dynamic tests showed that it is possible for the sensor frame to be loaded vertically upwards [2].

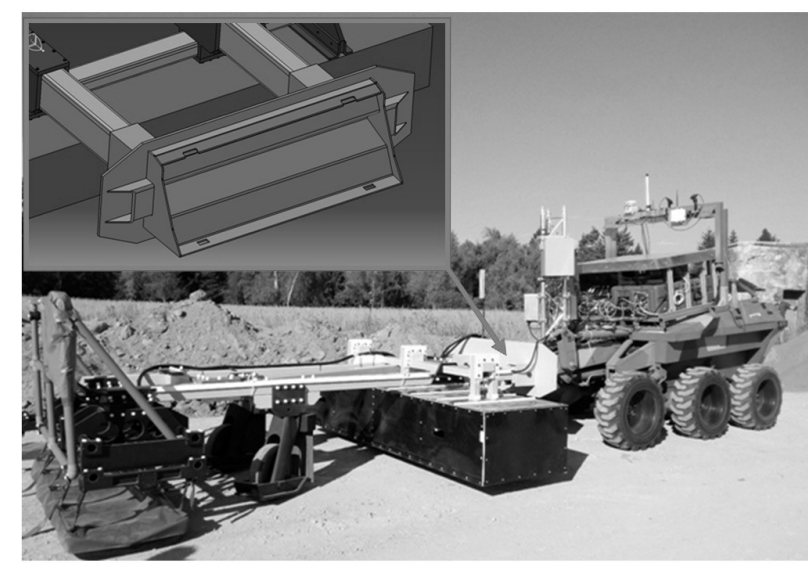

Fig. 7. View of Unmanned Ground Vehicle with mounted sensors frame

During the tests, sometimes the sensors frame was accidentally and uncontrolled disconnected from the quick coupler plate. This situation is highly undesirable. Therefore, it was necessary to modify the locking system in the quick coupler plate.

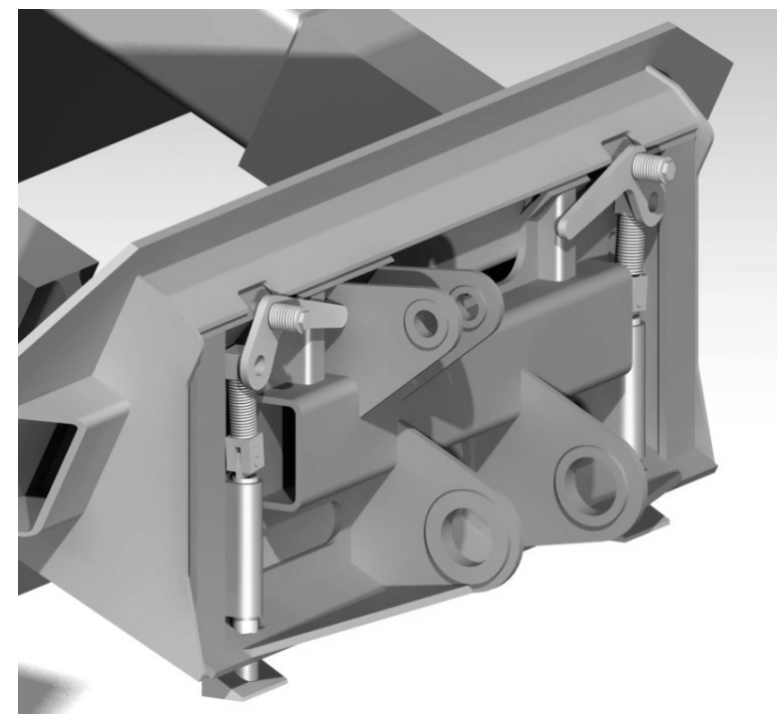

Fig. 8. View of the modernized quick-release plate locking system

As a result of the analysis, it was decided to change the length of the locking bolts. Because the conditions of the MUSICODE system do not require frequent disconnection of the sensors frame from the UGV, it was also decided to implement the mechanical screwed bottom bolt protection. The modernized locking system is shown in the Fig. 8 [2].

Conducting the necessary modification works on the platform (with particular emphasis on the GPR) required the collection of detailed requirements for individual detection subsystems. They concerned, inter alia, information such as:

- the number of components included in each detection system;

- external dimensions of each component;

- mass of each component;

- existing mechanical interfaces;

- electrical power of all components;

- existing electrical interfaces (location, wiring); 
- requirements for the location of elements with respect to metal elements and with respect to other sensors (Electromagnetic Compatibility).

The collected information enabled the development of a plan for the distribution of the main components of the detection system (Fig. 2).

It was decided that:

- the GPR, WD and MD will be attached to a support frame located on the front of the UGV;

- NLJD will be built directly on the platform (instead of a manipulator);

- all additional components (computers, measuring data processing elements, etc.) will be built above the UGV engine space;

- due to the insufficient power of the UGV alternator, it was decided to use an additional source of electricity to power the detection system components.

The decisions made allowed defining the available assembly spaces and conducting a preliminary functional - spatial - mass analysis. In the case of NLJD, the installation space at the front of the UGV (Fig. 9a) and the need to use existing mounting points (Fig. 9b.) are specified.

a)

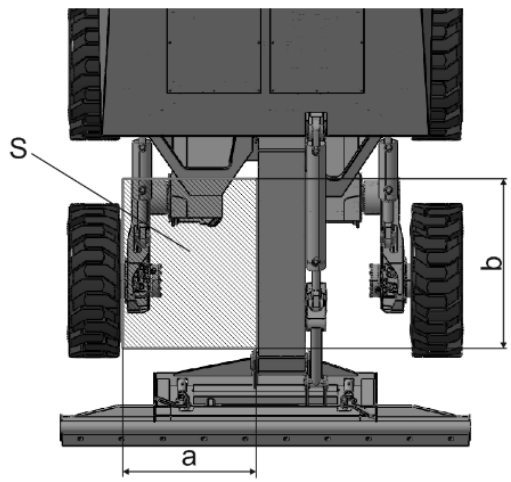

b)

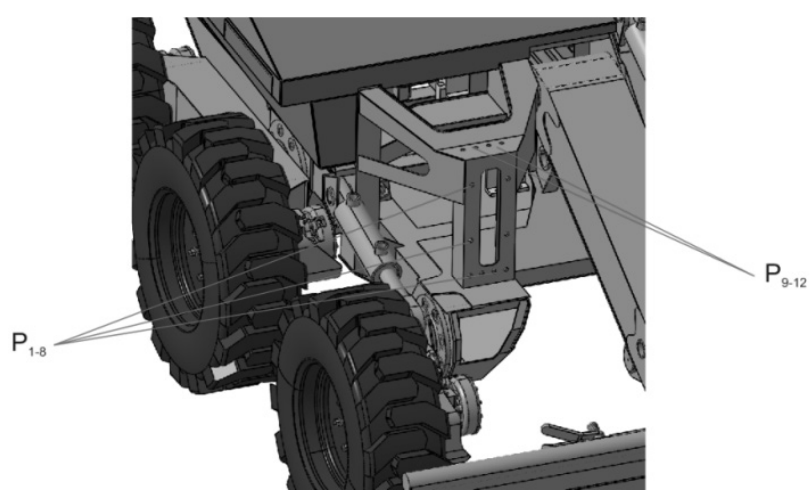

Fig. 9. View of the space selected for mounting NLJD (a) and existing mounting points (b)

The development of a support frame dedicated to $\mathrm{WD}, \mathrm{MD}$ and the GPR belonged to the area implemented by Military Institute of Technical Engineering (WITI). Then, the analysis of the possibility of placing computers, measuring data processing components and other components necessary for the detection system on the platform was carried out. The considerable size and mass of devices necessary for installa- tion on the UGV made it impossible to fit them using the current design possibilities. Particular emphasis was placed on the aspect of the area of visibility by teleoperation system cameras and not reducing the mobility of the robot. The analyzed areas are shown in the Fig. 10.

a)

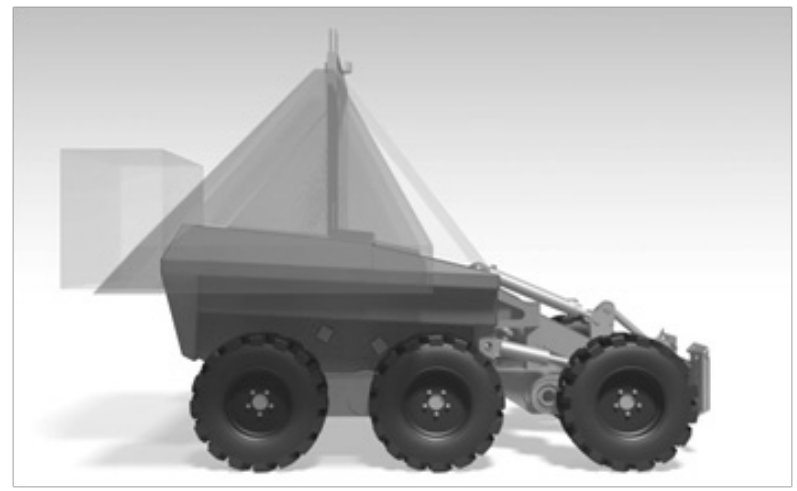

b)

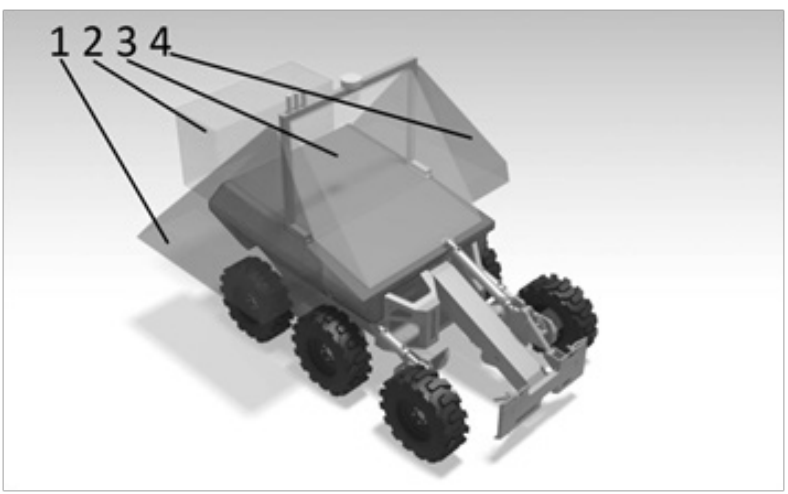

c)

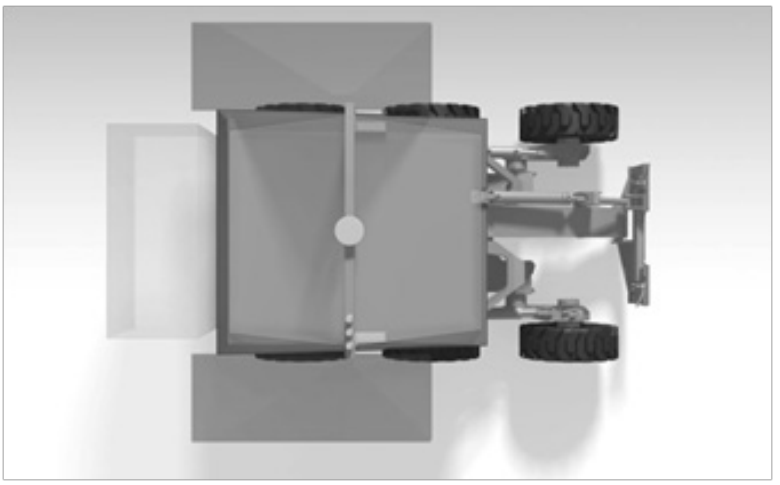

d)

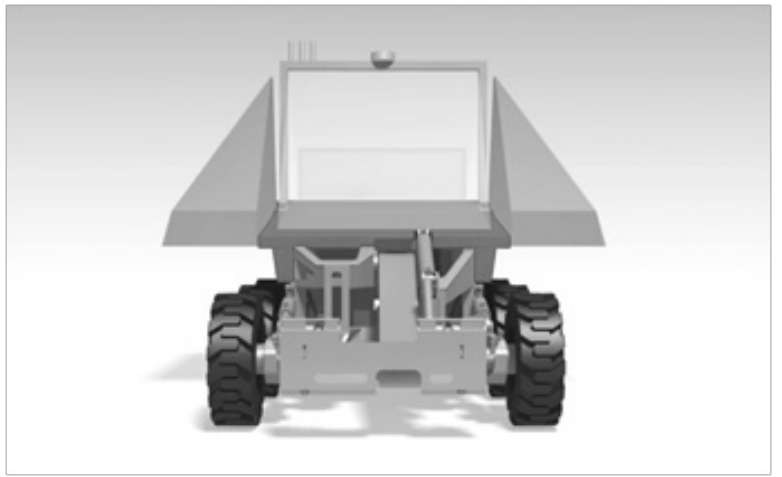

Fig. 10. Platform view with analyzed zones: a) side view, b) isometric view, c) top view, d) front view 
The area (Fig. 10) was divided into four zones: 1 - right, 2 - back, 3 - top and 4 - left. Zones 1 and 4 were excluded from the possibility of their use due to the presence of access points to the drive system, control, startup system, communication and the UGV fuel supply system in their areas. Due to the mass distribution on the modified UGV and its stability, zone 2 was reserved for the new power source. Only area 3 was prospective, but only in its front.

\section{Ground Penetrating Radar - Construction and Field Data Processing}

\subsection{Hardware}

The GPR detector is composed of 12 modules. Each module consists of one microwave ultra wideband transmitter and two independent receivers, so it contains two separate radar channels. It gives totally 24 channels that cover width of $3 \mathrm{~m}$ scanning path. The transmitter of each module illuminates the ground using central antenna and receivers record the radio wave echoes by two side antennas. The planar ultra-wideband Vivaldi-type antennas of all co-operating radio modules make linear array with $\lambda / 4$ offset $[12,13]$. The antenna array is shown in the Fig. 11.

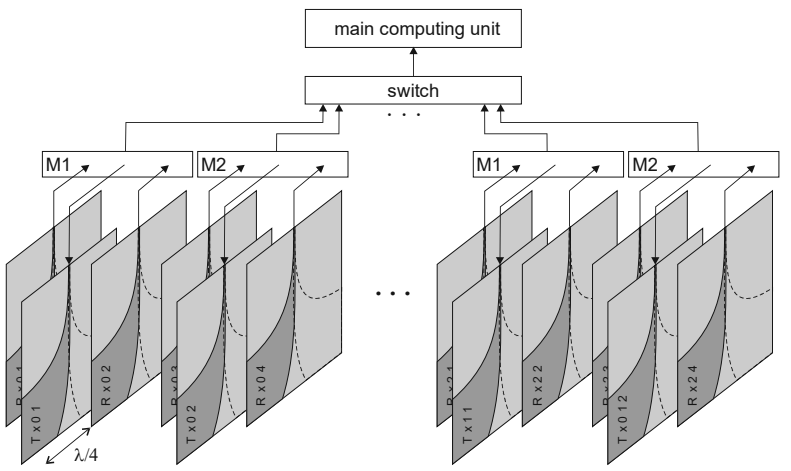

Fig. 11. Schematic view of antenna array and modules

All channels are time and spatial separated. The single channel diagram is shown in the Fig. 12. It consists of triangular waveform oscillator driving precise voltage control oscillator that generates signal of $1 \mathrm{GHz}$ bandwidth. Mixing it with the local oscillator signal $4 \mathrm{GHz}$ and its doubled frequency copy, one can obtain doubling of the bandwidth [14].

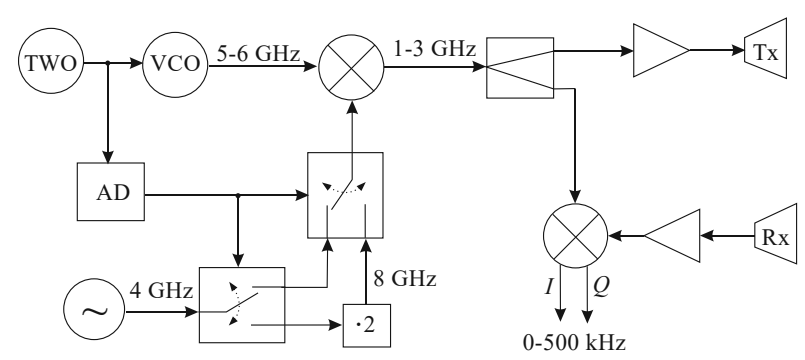

Fig. 12. Outlook of GPR bandwidth generation; TWO triangular waveform oscillator, VCO - voltage-controlled oscillator, AD - amplitude detector
The generated probing signal consists of two sub bands. The lower sub band is generated during an increase in voltage of TWO that drives VCO giving the frequency from 5 to $6 \mathrm{GHz}$; the upper sub band is making during a decrease TWO voltage and consequently frequencies from 6 to $5 \mathrm{GHz}$. In the first case, the signal from VCO is mixed with $4 \mathrm{GHz}$ and in the second case with $8 \mathrm{GHz}$ frequency. The switch of the frequencies follows the detection of minimum or maximum of TWO amplitude. As a result, the output signal is a sum of two sub bands 1-2 $\mathrm{GHz}$ and 2-3 GHz. The signal is coherent in a frame of sub bands. The transmitted signals are mixed with received echoes, so the frequency difference corresponds with depth of penetration and amplitude and phase gives an information about target existence and properties.

Each channel has the following key parameters:

- lower frequency $f_{\mathrm{L}} \sim 1 \mathrm{GHz}$;

- upper frequency $f_{\mathrm{H}} \sim 3 \mathrm{GHz}$;

- stepped frequency continuous wave modulation (SFCW);

- number of frequencies $N=500$;

- value of frequency step $\Delta f=4 \mathrm{MHz}$;

- phase shift resolution $\Delta \phi=0.1^{\circ}$;

- time resolution $t_{\mathrm{r}}<0.4 \mathrm{~ns}$;

- maximum power of signal $20 \mathrm{dBm}$.

The intermediate frequency signal is next pre-processed using dedicated fast electronic field-programmable gate array (FPGA) structures that provide the resulting data over standard protocol to the high-level software.

The internal organisation of single module is shown in the Fig. 13.

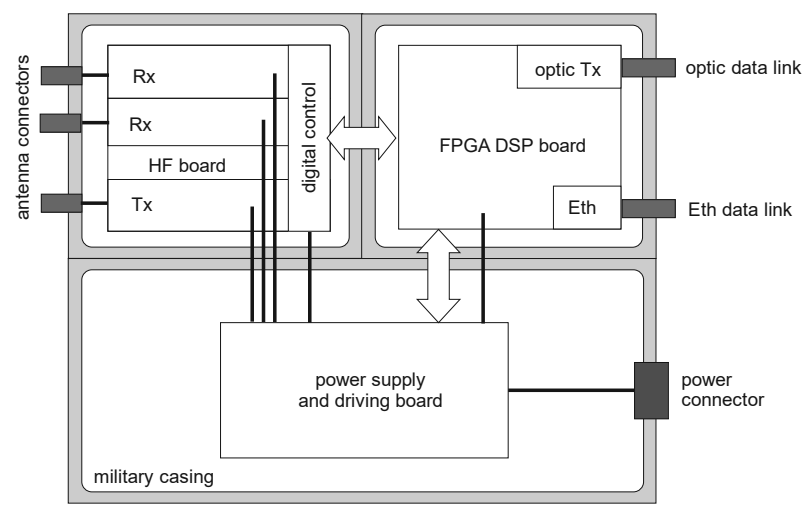

Fig. 13. Internal organization of single module

The hardware modules and antenna array are mounted inside dedicated metal-free casing. The space-time data necessary to mark the detections are generated by separate GPS system that send data markers for all collaborating detectors.

\subsection{Signal Processing}

The digitized $I Q$ signals are submitted to the discrete Fourier transform (DFT) FPGA module. The transform in the such kind of radar plays the role of the range compression procedure. An exemplary spectrum of the signal is illustrated in the Fig. 14. It may be observed that the information on the ground's re- 
flectivity is contained in the transform domain. The frequency domain has been normalized to the sampling frequency range of the transform samples for positive frequencies. The GPR is designed to detect objects buried in shallow layers of the ground, therefore it does not require signals representing echoes from the whole range. Due to this only a selected set of the DFT samples is stored in the system and sent to the signal processing unit.

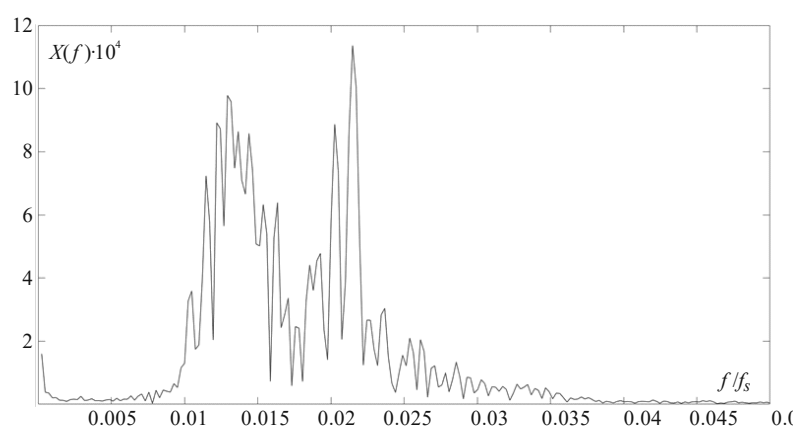

Fig. 14. An exemplary spectrum obtained in chosen channel

The signal processing procedure starts with sorting of vectors corresponding to appropriate receiving channels, and aligned according to the time stamps obtained from GPS. The block diagram of the procedure chain is shown in the Fig. 15.

Due to the comparatively fast movement of the carrier platform on rough ground surface along the tested route, the whole GPR modules and antennas strongly vibrate. This induces fluctuations of the received signals. It requires the filtration of those fluctuations in the slow time domain and, as the vibrations are of higher frequency than the changes in the ground that are of interest, a low-pass filter is applied with a $-3 \mathrm{~dB}$ cut-off relative frequency of $0.12[\mathrm{~Hz} /$ $\mathrm{Hz}]$ that removes the vibrations.

In the Fig. 16 an exemplary input signal and its filtered version is shown. a)

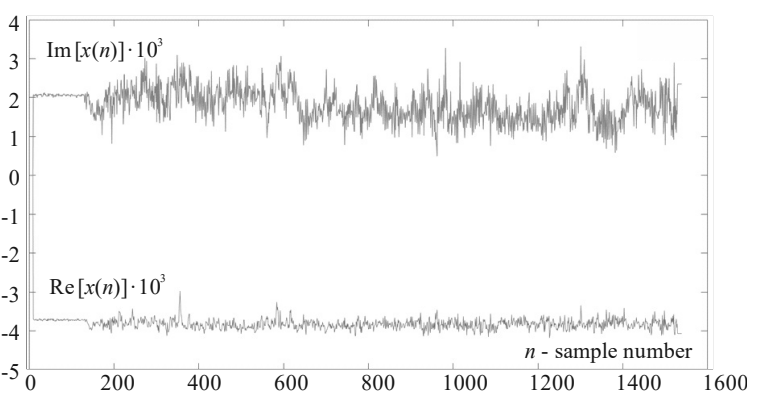

b)

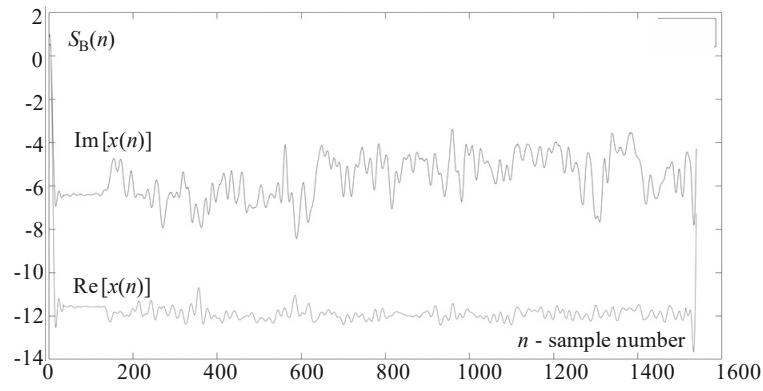

Fig. 16. An exemplary signal before (a) and after lowpass filtration (b)

The filtered signals are next weighted in order to equalize the amplification of the particular channels. The equalization is based on measurements of the mean magnitude of the noise signals in each channel. During the measurement, as has already been indicated, each module sends two successive linear frequency modulated signals in two separate sub-bands, a lower and a higher one. The combined bandwidth of the two sub-bands is equal to $2 \mathrm{GHz}$, and the sum of the signals from both the sub-bands allows to achieve the full range resolution of the GPR sensor. Therefore, the filtered and weighted complex signals are combined giving 24 signals that create a complete complex 3-D image of the ground's reflectivity.

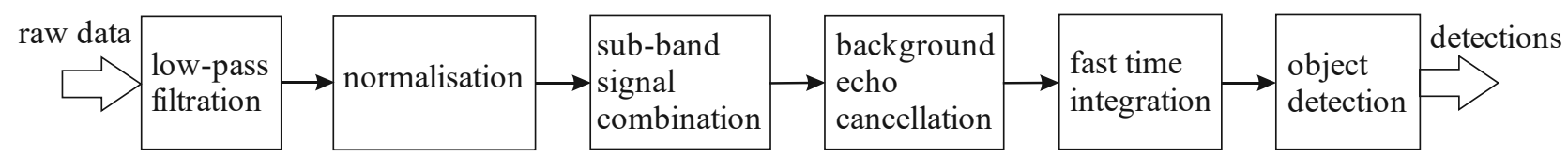

Fig. 15. Block diagram of the signal processing chain 


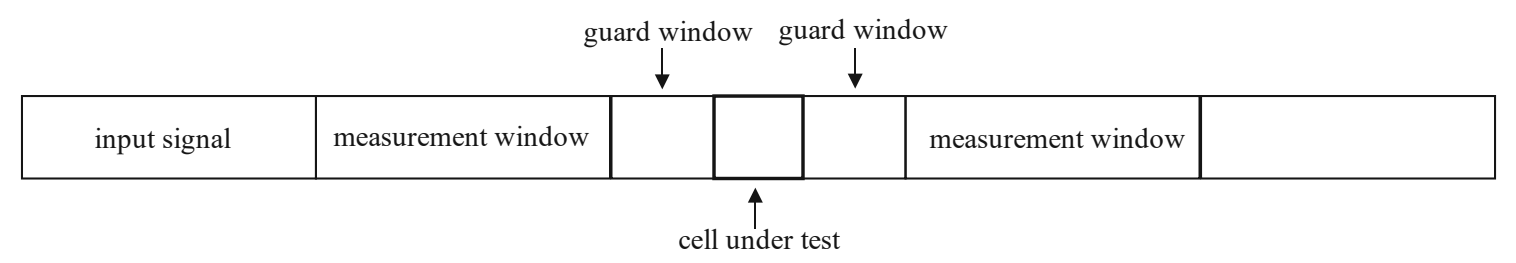

Fig. 17. Distribution of the measurement windows

In order to extract the in homogeneities of the ground, that may appear dangerous objects, a background removal procedure is applied. This procedure bases on averaging the level of background echo signals around the tested chosen cell and subtracting it from the analyzed signal. The measurement is done by averaging the signal levels in two windows in the left and right neighborhood of the cell under test (CUT). In order to separate the measurement from the signal level in the CUT, two guard windows around the CUT are applied. There is shown in the Fig. 17 the distribution of the measurement windows in the algorithm.

There are many variants of this procedure and majority of them base on averaging the background signals in measurement windows, there are also algorithms that base on the principle of a kind of a median filter that performs a sorting in the two measurement windows, and then finds the value on $n_{0}$-th cell in the sequence taking it as the estimate of the background signal level. Those algorithms are very similar to the well known from classic radar technology CFAR (Constant False Alarm Ratio) algorithm with the general difference that the estimate of the background is not compared to any thresholds, due to the fact that the GPR should detect sudden lowering of the echo levels as well as peaks $[15,16]$. The implemented algorithm is illustrated in the Fig. 18.

The background elimination algorithm consists of two main steps:

1. For each $n$-th cell the estimates $C_{L}$ i $C_{R}$ of the background echo signal level in two windows (left and right) are computed, each window has the length of $N$, the edge of each window is at the distance of $G$ cells from the CUT position;

$$
\begin{aligned}
& C_{L}(n)=\sum_{k=n-N-G}^{n-G-1} S_{B}(k), \\
& C_{R}(n)=\sum_{k=n+G}^{n+N+G} S_{B}(k),
\end{aligned}
$$

2. The estimates are added up and divided by $2 N$, subsequently the average is subtracted from the signal in the CUT, the difference is stored in the $n$-th cell of the output signal:

$$
S_{B E}(n)=S_{B}(n)-\frac{C_{L}(n)+C_{R}(n)}{2 N}
$$

The next procedure of the signal processing chain is the signal integration in the fast time direction (towards the ground depth). The objects sought by the GPR, i.e., improvised explosive devices, in order to gain a proper effect, for example destruction of an armored vehicle, need to be of significant size. This means that their echo will dwell more than one range cell. In order to improve the signal-to-disturbance ratio an integration along the range direction is performed:

$$
S_{\text {Int }}(p)=\sum_{k=p-1}^{p+1} S_{B}(k)
$$

where $p$ is the number of integrated range cells.

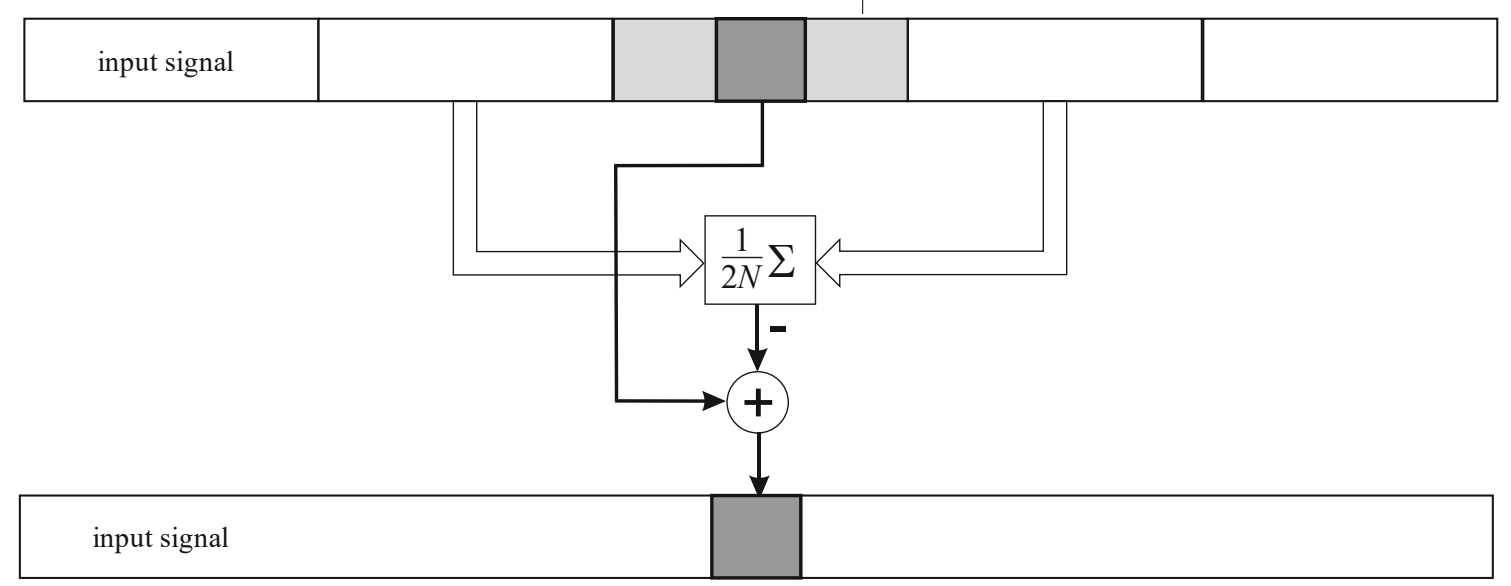

Fig. 18. Block diagram of the background elimination algorithm 
In the Fig. 19 there is presented the signal before and after the background elimination procedure.

a)

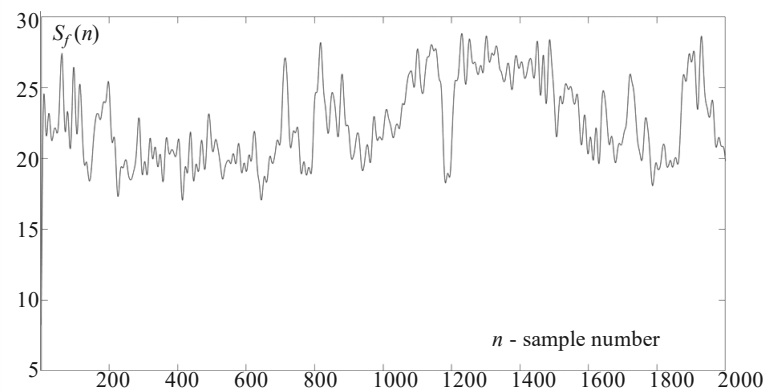

b)

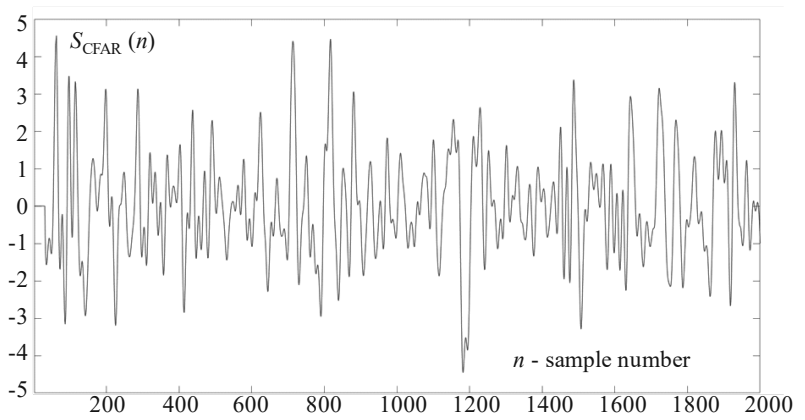

Fig. 19. The signal before (a) and after the background elimination algorithm (b)

After the integration a comparison of the signal with a threshold is performed and the detection report is generated. The report in JSON format is next sent to the fusion unit gathering information of all sensors of the platform. The additional subroutine allows to preview the detections. In the Fig. 20 there is shown an exemplary imaging generated using the subroutine.

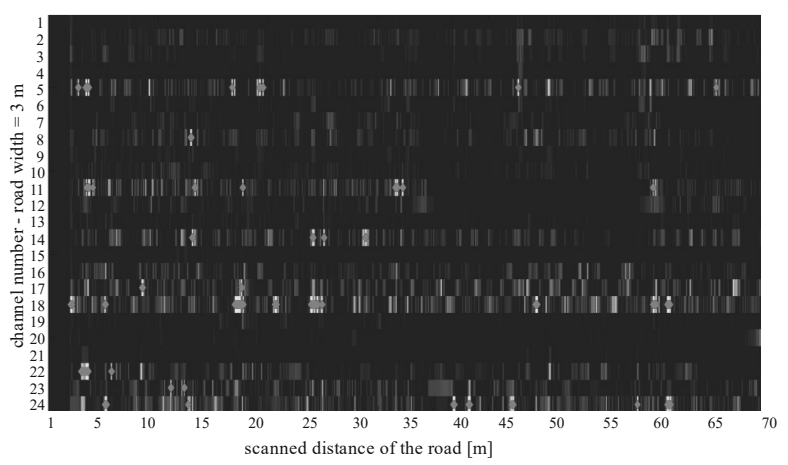

Fig. 20. An exemplary imaging of the detections at the distance of $70 \mathrm{~m}$. The dots indicate suspicious objects

The detection report contains also false alarms that should be eliminated in a final fusion process.

\section{Conclusion}

The described UGV design and the results of GPR field data processing being one of the final results of the
MUSICODE project allowed to find the answer to the research question: there is an impact of using Ground Penetrating Radar to be mounted on Unmanned Ground Vehicle to detect improvised explosive devices (IEDs) on the UGV construction and the GPR results. The changes in the structure of UGV allowed to fulfil the requirements to be the carrier for IED detection sensors but with some limitations resulting from the basic structure of current version of the UGV. The UGV used in the project never demonstrated a driving speed of $30 \mathrm{~km} / \mathrm{h}$ which was one of the goals. It is possible to achieve such a UGV speed by making design changes resulting from a change in the approach to the platform capability based on additional sources of information.

The lessons learned knowledge can be implemented in the continuation works within this IED detection area. Further research should be focused on new design of UGV dedicated for the carrier of different sensors that should be used in IED detection missions and on development of new versions of the sensors with particular attention on GPR.

The main goal of the new project should be development of a new generation of sensors to explore the possibilities of increasing the detection and identification of IEDs in complex tactical and operational conditions, and to develop a new solution of UGV.

The specific objectives of the new project in the field should be:

a) examining the possibilities of integrating early warning and remote detection systems on one reconnaissance platform.

b) examining the possibility of direct cooperation of a group of robots for the detection and protection of convoys - early warning / remote detection as well as confirmation and identification unification of the pace of operations;

c) development of swarm technology and miniaturization of smaller and lighter sensors [18];

d) development of a new multi-task base platform that can act as a carrier of systems, in particular detection task;

e) development of the suspension and control system (voice control) of the UGV as well as its executive mechanisms, including innovative manipulators with haptic control.

f) development of a manipulator solution enabling confirmation and identification of IEDs placed in the ground or masked with heavy objects (necessary load capacity of 50-80 kg at a range of 3-4 m);

g) development of a manipulator solution enabling rapid inspection of heavy goods vehicles and culverts without leaving the robot off the road crown;

h) development of new control systems for inspection and intervention manipulators (with haptic and vector control);

i) examining the possibilities of expanding the GPR system detection capabilities to detect of nonlinear junctions located on the route and detection of metal clusters (possibility of taking over the role of an inductive metal detector); 
j) examining the possibility of mechanical stabilization of the position of GPR antenna.

The new UGV should be modular and open architecture to be better adaptable to different missions including IED detection missions. There should be improved among others the suspension system to be useful for carrying the new generation of sensors including GPR, manipulators to be able to fulfil the requirements coming from the new generation of CONOPS.

\section{ACKNOWLEDGEMENTS}

This work was supported by the European Defence Agency under PROGRAMME ARRANGEMENT (PA) No B 1465 GEM3 GP concerning the "IED Detection Programme" (IEDDET-programme).

\section{AUTHORS}

Piotr Szynkarczyk - ŁUKASIEWICZ Research Network - Industrial Research Institute for Automation and Measurements PIAP, Al. Jerozolimskie 202, 02-486 Warsaw, Poland.

Józef Wrona* - ŁUKASIEWICZ Research Network Industrial Research Institute for Automation and Measurements PIAP, Al. Jerozolimskie 202, 02-486 Warsaw, Poland, Email: jozef.wrona@piap.lukasiewicz. gov.pl.

Mateusz Pasternak - Military University of Technology, gen. Sylwestra Kaliskiego 2, 00-908 Warsaw 46, Poland.

Arkadiusz Rubiec - Military University of Technology, gen. Sylwestra Kaliskiego 2, 00-908 Warsaw 46, Poland.

Piotr Serafin - Military University of Technology, gen. Sylwestra Kaliskiego 2, 00-908 Warsaw 46, Poland.

*Corresponding author

\section{REFERENCES}

[1] “The 'MUSICODE' Project". PIAP, https://piap. lukasiewicz.gov.pl/en/research-projects/themusicode-project/. Accessed on: 2021-12-22.

[2] "Report on UGV with integrated sensors", EDA, 2020.

[3] A. A. Faust, C. J. de Ruiter, A. Ehlerding, J. E. McFee, E. Svinsås and A. D. van Rheenen, "Observations on military exploitation of explosives detection technologies". In: R. S. Harmon, J. Holloway and J. T. Broach (eds.), Proc. Volume 8017, Detection and Sensing of Mines, Explosive Objects, and Obscured Targets XVI, 2011, 10.1117/12.886391.

[4] S. C. Spry, A. R. Girard and J. K. Hedrick, “Convoy protection using multiple unmanned aerial ve- hicles: organization and coordination". In: Proc. of the 2005, American Control Conference, 2005, 3524-3529, 10.1109/ACC.2005.1470519.

[5] S. M. Silva, J. D. Gamarra, C. A. Hernandez and J. F. Osma, "Design and fabrication of a sensor for explosives as a first step to an IED detection device". In: 2014 IEEE 9th IberoAmerican Congress on Sensors, 2014, 1-4, 10.1109/IBERSENSOR.2014.6995515.

[6] M. Garcia-Fernandez, A. Morgenthaler, Y. Alvarez-Lopez, F. Las Heras and C. Rappaport, "Bistatic Landmine and IED Detection Combining Vehicle and Drone Mounted GPR Sensors", Remote Sens., vol. 11, no. 19, 2019, 10.3390/ rs11192299.

[7] J. Czarnowski, A. Dąbrowski, M. Maciaś, J. Główka and J. Wrona, "Technology gaps in Human-Machine Interfaces for autonomous construction robots", Autom. Construct., vol. 94, 2018, 179-190, 10.1016/j.autcon.2018.06.014.

[8] A. Bouhraoua, N. Merah, M. AlDajani and M. Elshafei, "Design and implementation of an unmanned ground vehicle for security applications". In: ISMA'10 - 7th International Symposium on Mechatronics and its Applications, 2010.

[9] J.Łopatka, T.MuszyńskiandW.Polis, “Modułowe lekkie bezzałogowe platformy lądowe wsparcia”, Szybkobieżne Pojazdy Gąsienicowe, vol. 45, no. 3, 2017, 99-113, (in Polish).

[10] P. Klinkhachorn, A. S. Mercer, U. B. Halabe and H. V. S. GangaRao, "Unmanned Ground Vehicle for Autonomous Non-Destructive Testing of FRP Bridge Decks". In: AIP Conference Proc., vol. 894, 2007, 1723-1730, 10.1063/1.2718172.

[11] A. Bartnicki, M. J. Łopatka, L. Śnieżek, J. Wrona and A. M. Nawrat, "Concept of Implementation of Remote Control Systems into Manned Armoured Ground Tracked Vehicles". In: A. M. Nawrat (ed.), Innovative Control Systems for Tracked Vehicle Platforms, vol. 2, 2014, 19-37, 10.1007/978-3-319-04624-2_2.

[12] M. Pasternak, "Lambert W function application for construction of antipodal Vivaldi-type antenna". In: 2018 14th International Conference on Advanced Trends in Radioelecrtronics, Telecommunications and Computer Engineering (TCSET), 2018, 624-627, 10.1109/TCSET. 2018.8336279.

[13] Nurhayati, G. Hendrantoro and E. Setijadi, "Mutual coupling and radiation pattern of Vivaldi antenna with slit". In: Proc. of the 3rd International Conference on Communication and Information Processing - ICCIP '17, 2017, 296-300, 10.1145/3162957.3163056.

[14] M. Pasternak and P. Kaczmarek, "Continuous wave ground penetrating radars: state of the art". In: XII Conference on Reconnaissance and Electronic Warfare Systems, vol. 11055, 2019, 84-89, 10.1117/12.2524524. 
[15] M. Kronauge and H. Rohling, "Fast Two-Dimensional CFAR Procedure", IEEE Transactions on Aerospace and Electronic Systems, vol. 49, no. 3, 2013, 1817-1823, 10.1109/ TAES.2013.6558022.

[16] M. Labowski, P. Kaniewski and P. Serafin, "Motion Compensation for Radar Terrain Imaging Based on INS/GPS System", Sensors, vol. 19, no. 18, 2019, 10.3390/s19183895.

[17] "EDA IED Detection Programme (IEDDET)". M. Kalbarczyk, http://www.irsd.be/website/ images/images/Activites/Colloques/presen tation/2016-05-17/05-Mr-Marek-KALBAR CZYK.pdf. Accessed on: 2021-12-21.

[18] MUSICODE consortium and PMG, "MUSICODE D.1.2 - Project Final Report”, B 1465GEM3 GP, 2020. 